\title{
Factores que influyen en el deportista de rendimiento para avanzar en su práctica en el patinaje modalidad carreras
}

\section{Factors that influence the performance athlete to advance in their practice in the modality skating races}

\author{
MARTÍNEZ-MONROY, Sorayda ${ }^{1}$ \\ MURCIA, Immer O. \\ ALVARADO-TORRES, Jhon E. ${ }^{2}$
}

\begin{abstract}
Resumen
El presente artículo es producto de investigación desde una perspectiva Bio-ética, con enfoque cualitativo y alcance descriptivo que permitió identificar factores que influyen en el deportista de rendimiento para avanzar en su práctica en el patinaje modalidad carreras, afiliados a clubes y/o escuelas de la localidad de Kennedy de la ciudad de Bogotá, D.C. Se realiza contexto sobre deporte y adolescencia, incluyendo a los actores que intervienen en el proceso deportivo (padres de familia, entrenadores, academia y clubes de patinaje).

Palabras clave: patinaje de carreras, rendimiento deportivo, factores, influencia
\end{abstract}

\begin{abstract}
This article is the product of research from a Bio-ethical perspective, with a qualitative approach and descriptive scope that allowed identifying factors that influence the performance athlete to advance in their practice in skating modality races, affiliated with clubs and / or schools of the Kennedy locality of the city of Bogotá, DC Context on sports and adolescence is carried out, including the actors involved in the sports process (parents, coaches, academy and skating clubs).

key words: racing skating, sports performance, dropout, factors, influence
\end{abstract}

\section{Introducción}

Todo proceso de formación deportiva implica toma de decisiones con relación a un proyecto de vida en el que se involucran en primer termino los deportistas en formación y alrededor suyo padres de familia, profesionales de ciencias aplicadas al deporte, profesores, directivos y entorno social. Por tanto, al conformar un ideal de proyección deportiva, se hace necesario considerar el análisis de factores psicosociales, administrativos y técnico-deportivos para evitar elecciones basadas únicamente en los logros deportivos alcanzados a muy temprana edad y así avanzar en su práctica deportiva.

En este sentido, muchos de esos proyectos de vida deportiva se han visto truncados debido a diversos factores, especialmente los relacionados con aspectos económicos, falta de tiempo por necesidad de atender otras

\footnotetext{
${ }^{1}$ Docente investigadora. Administración Deportiva. Universidad Distrital Francisco José de Caldas. smartinez@udistrital.edu.co

2 Profesor titular - Tiempo completo, Facultad de Medio ambiente y recursos naturales. Universidad Distrital - Francisco José de Caldas. jealvaradot@udistrital.edu.co
} 
prioridades, la desmotivación ocasionada por múltiples causas y en algunos casos inadecuados procesos administrativos y pedagógicos llevados a cabo en las escuelas y clubes de iniciación deportiva.

\section{Factores que influyen en el deportista de rendimiento para avanzar en su práctica en el patinaje modalidad carreras}

Lo anterior se explica en la dinámica actual del deporte y la actividad física, donde la interacción de todos estos factores agregan valor importante para el desarrollo humano a través de la organización de diferentes disciplinas deportivas. A partir de allí, es común encontrar escuelas y clubes de formación deportiva, donde las posibilidades para un deportista de practicar su disciplina tanto a nivel aficionado o de alto rendimiento se incrementan. Por ejemplo, en la ciudad de Bogotá, capital de Colombia, se encuentran avaladas por el Instituto Distrital de Recreación y Deporte según lo relacionado tabla anexa en su sitio web (IDRD, 2020) 151 escuelas de formación deportiva deportiva avaladas al 31 de agosto de 2020. No obstante, existen en las 20 localidades del Distrito Capital escuelas y clubes no avaladas que superan la cifra institucional.

En esta línea, si bien existe amplia oferta de modalidades y categorías deportivas por edades qué garantizan a clubes y escuelas el ingreso a procesos de formación o perfeccionamiento deportivo, la presente investigación encuentra también resultados notables en cuanto a abandono en el transcurso del desarrollo de las carreras deportivas.

En este sentido, algunos factores que inciden en la deserción de un proyecto deportivo son los compromisos académicos del atleta, la falta de tiempo, el aburrimiento, la pereza y la desmotivación entre otros. De igual forma, como lo mencionan Cecchini Estrada, Echevarría Castillo, y Méndez Giménez, (2003) se debe considerar que un gran número de jovenes abandona su práctica deportiva, dado que se inicia en ella por experimentar para posteriormente tomar la decisión de continuar o no y, atribuye esto a la naturaleza inconformista y curiosa que presentan los niños y jóvenes entre 11 y 15 años de edad. Además, mencionan en sus estudios la edad de 14 años como el acmé donde los jóvenes presentan mayor abandono de sus prácticas deportivas, lo cual representa aproximadamente el 35\% de deserción anual en las escuelas y clubes de formación deportiva inicial. (Macarro Moreno, 2010) adicionalmente señalan evidencias de diversos estudios que ponen de manifiesto un problema de abandono de la práctica de actividad físico-deportiva a medida que avanza la edad en los jóvenes, y muestran una tendencia hacia la adquisición de estilos de vida sedentarios y poco saludables.

Es en este contexto, no solo se identifican los factores que influyen en la continuidad del deportista de rendimiento en la práctica del patinaje de carreras sino que, se reflejan aspectos que condicionan la deserción de procesos de formación o perfeccionamiento deportivo, relacionados en importante medida con el entorno psicosocial del patinador y otros más, que se enmarcan en características de la escuela o club de formación deportiva a la que se encuentran adscritos.

\subsection{Análisis bibliométrico}

La investigación parte del análisis bibliométrico en el periodo comprendido entre 1996 y 2020, se identifican en Scopus siete documentos, de los cuales, el $14,3 \%$ equivalen a revistas y el $85,7 \%$ son artículos. Cabe resaltar, que tan solo existe un artículo de investigación de Colombia; dos documentos de España, uno de Austria, uno de Brasil y uno de Canada. Las principales fuentes de información son: Revista internacional de morfo fisiología, Cuadernos de psicología deportiva, Revista ocio y sociedad, Archivos generales de medicina y revista Movimiento de la Facultad de Educación Física de la Universidad Federal do Rio Grande do Sul.

El análisis bibliométrico no arrojó documentos similares al objeto de investigación pero en relación al vocablo patinaje presenta áreas de conocimiento incidentes, producto de la actividad investigativa: psicología 9\%, ciencias sociales $27 \%$, ciencias naturales $9 \%$, profesionales de la salud $27 \%$ y medicina $28 \%$. 
Igualmente, se evidencia un crecimiento cercano a 1.500 publicaciones al año, entre 2000 y 2020 en torno a citación de fuentes de información para producción de conocimiento científico acerca del tema patinaje.

El análisis indicó mayor consulta en la Revista Internacional de morfo fisiología, la cual tiene un punto de inicio en el año 2006 con tan solo 3 citaciones pero que para el año siguiente aumentaría a 24 y tendría un salto exponencial a 131 para el año 2009, 240 en el 2010 y con un crecimiento aproximado de 200 citaciones por año, hasta llegar al 2020 a 1.080 citaciones. En comparación con otras fuentes de información como Cuadernos de psicología del deporte con 417 citaciones, 200 de la Revista Ocio y sociedad, 234 de Nuevas tendencias y 79 de Cuadernos de medicina del deporte; se amplia el número de citaciones tal vez, por su carácter internacional.

Sin embargo, es curioso que exista un alto índice de citación, pero una baja productividad científica reflejada en publicación de artículos. Por consiguiente, a partir de la relación de citas por artículo publicado la presente investigación permite contribuir al vacío en conocimiento y a la apropiación social del mismo desde la identificación y, proporciona valor adicional para comparar y evaluar revistas científicas con relación al deporte. Para esto, se tuvo en cuenta la comparación métrica de Cite Score basado en el número de citas recibidas por documentos publicados en una revista en los últimos 4 años, dividido por el número de documentos publicados en ese mismo periodo.

\subsection{Soporte conceptual}

El estudio se enmarca en el deporte del alto rendimiento, el cuál se inicia en edades tempranas y cada día es más exigente con los atletas, las cargas de volumen e intensidad son mayores pensando en la victoria más no en la deserción. Al respecto, Pancorbo \& Blanco (1990) plantean que las cargas de entrenamiento deportivo deben ser adecuadas para que los deportistas jóvenes puedan rendir, entendiendo que el niño se encuentra en desarrollo y a cada etapa de crecimiento le corresponden cargas que deben ser respetadas. En ese sentido, según Navarro Valdivielso, (2004), el entrenamiento de niños y jóvenes es posible y recomendable siempre que se ajuste a los principios de adaptación individual determinados por edad y sexo; lo cual implica considerar en todo momento las posibilidades biológicas, el talento, la motivación y la disposición del niño para lograr resultados óptimos.

Es por ello, que una correcta planificación y metodología del entrenamiento a largo plazo, desde el conocimiento de la estructura morfofisiológica de cada atleta, su edad biológica y la etapa de crecimiento entre otras cualidades básicas, permiten evitar la deserción por desgaste físico y mental en practicantes. Por tanto, en concordancia con la clasificación de formación atlética propuesta por Barzi (1987) y citadas por Pancorbo \& Blanco (1990); las edades entre 6 y 11 años son consideradas parte del proceso de iniciación deportiva; el estadio de especialización deportiva o juvenil, desde los 12-13 años hasta los 16 o 17 años y alto rendimiento o adulto de 17 años en adelante. (Pancorbo \& Blanco, 1990).

Por otro lado, la identificación de un deportista de alto rendimiento deportivo consiste en predecir si un joven podrá desarrollar el potencial de adaptación al entrenamiento y su capacidad de aprendizaje técnico, para emprender las posteriores etapas del entrenamiento. De acuerdo con Benítez Moya (2011) esta identificación se entendería como una fase del proceso de determinación de las capacidades del rendimiento que una persona deberá tener a fin de poder alcanzar un alto nivel en el futuro. En la identificación temprana de deportistas, Leiva De Antonio, (2017), afirmó que los niños normales nacen con un vasto repertorio de posibilidades motoras que les confiere un amplio espectro de reflejos que implican los sentidos de postura y movimiento, y a medida que progresa su desarrollo la necesidad de movimiento toma la forma de juego para canalizarse más tarde en actividades deportivas. 
En ese sentido, la identificaión de un niño para la consecución de altos logros suele tener en cuenta aspectos más allá de la perfecta ejecución técnica; por ejemplo, involucra no solo estudios genéticos sobre características de los padres sino, condiciones medioambientales óptimas qué favorecen el desarrollo del potencial de los deportistas. Entre los factores medioambientales se cuenta el entrenamiento, la competencia, los implementos deportivos, las condiciones nutricionales, el estado de salud, la vida intrafamiliar, entre otros. Desde esta perspectiva, se podría afirmar que se nace con las condiciones fisiológicas, antropométricas, psicológicas, motoras o técnicas para rendir mejor en un deporte que en otro.

Sin embargo, en la formación de deportistas no todos los niños asimilan con rapidez y de la misma forma las técnicas deportivas para el desarrollo de capacidad física y mental; o, simplemente no están capacitados para alcanzar los mismos resultados en la competición. Por tanto, aquellos que se destacan en una modalidad deportiva por su capacidad de asimilación y rendimiento se les denomina talentos (Villamarin Menza, 2015). Para Volkov y Filin (1988) citados por Villamarin Menza (2015), la selección deportiva es un sistema de medidas organizado metodológicamente que incluyen los factores pedagógicos, psicológicos, sociológicos y médico biológicos de investigación, base de detección de las capacidades de niños, adolescentes y jóvenes para especializarse en una determinada modalidad deportiva. Es así como, la preparación de reservas deportivas implica una rigurosa continuidad de las tareas, medios y métodos de entrenamiento de los niños, el incremento sostenido del volumen de entrenamiento general y especial, el incremento progresivo del volumen e intensidad de la carga y la aplicación del principio de gradualidad en la aplicación de la carga de entrenamiento y competición.

Para Hahn 1998 citado por Villamarin Menza, (2015), existen tres tipos de talentos: talento motriz general, aquella persona capaz de aprender rápidamente una gran cantidad de movimientos; talento deportivo, deportistas que además de aprender rápido las habilidades motoras, están predispuestos a someterse a programas de entrenamientos; y el talento específico deportivo, hace referencia a aquellos deportistas que requieren condiciones físicas y psíquicas para alcanzar rendimientos específicos en un deporte. De igual forma, otro factor incidente en la continuidad o deserción de procesos de alto logro deportivo por parte de los jovenes en iniciación deportiva y durante el desarrollo posterior es la influencia del entorno social como variable importante del rendimiento atlético. (Villamarin Menza, 2015).

En ese sentido, se encuentran datos relacionados con el éxito deportivo y su codependencia ante una diversa interacción de factores hereditarios y ambientales; por tanto, ambas variables son relevantes y deben ser suficientemente contempladas en la formación de un deportista. Entre las consideraciones sociológicas están implícitas variables como la preparación, la influencia de otros significativos, el ambiente, la cultura, el estado socioeconómico, la movilidad social, la inteligencia e incluso la suerte. (Capetillo Velasquez, 2011)

Por consiguiente, dependiendo tanto del entorno geográfico como del medio ambiente social donde se encuentre e interactúe el niño, tendrá mejores y mayores oportunidades de desarrollo no sólo social e intelectual sino también motriz. Al respecto, Capetillo Velásquez (2011) citando a Richardson y Reilly (2001), consideró que, es imperativo analizar en un deportista la diversidad de variables sociológicas tales como: la preparación, el ambiente, la cultura, el estado socioeconómico, la movilidad social, la inteligencia, incluso la suerte; de tal manera que un jugador de fútbol en proceso de formación sin duda necesita para su óptimo desarrollo del influjo positivo del medio social y ambiental. Una de las variables sociológicas incidentes en el logro y desempeño del deportista es el entorno familiar. Al respecto, algunos teóricos de la educación han resaltado la influencia sociocultural en la estructuración de la personalidad y el carácter, los cuales son indispensables para la actuación exitosa en el deporte. 
Las posibilidades que el niño o joven practique un deporte y se mantenga activo es más probable si esa decisión esta auto determinada y no determinada por otro, como sucede en muchos casos, que está direccionada por los adultos; el rendimiento llega a ser formativo si el joven conoce sus posibilidades y límites, le ayuda a experimentar su potencial y a reconocer el crecimiento de sí mismo (Villamarin Menza, 2015)

Es por ello, que la influencia de padres sobre los jovenes deportistas en muchos casos es notoria y determinante para el proyecto de vida deportivo. Para Villamarin Menza, (2015) citando a Dietrich Martin y Cols (2001), los factores más reveladores que condicionan al joven deportista son la familia (padres), los amigos, la escuela, la formación profesional y las ofertas de los clubes deportivos; de igual manera, reconocen también a la familia como el agente mediador de mayor influencia en la vida del deportista y agregan que éstos proporcionan el ambiente social primario donde puede desarrollar su identidad, autoestima y motivación para tener éxito en el deporte. En un estudio realizado por Morillas Cabezas en Argentina (2001), se observó que existe una relación directa con diferencias significativas entre aquellos jóvenes deportistas que gozan de un ambiente familiar óptimo y aquellos que no, presentando los primeros unos niveles elevados de autoconfianza, superiores a los presentados por los segundos.

Otra de las variables sociológicas que se considera como importante en el logro y desempeño del deportista es el ambiente en el cual se desenvuelve su práctica de formación deportiva. En este sentido, en un estudio de Nuviala Nuviala, García Montes, Ruiz Juan, \& Jaenes Sánchez, (2006), en el que se buscaba establecer las relaciones entre el modelo deportivo escolar y el abandono de los deportistas; encontró que cuando el modelo organizativo se orienta únicamente hacia la competición y hacia una sola modalidad deportiva, donde el fin principal es la victoria, se exige a los participantes un elevado coste físico y psicológico, esto derivará en una situación de frustración para un gran número de los deportistas que comienzan, produciendo un abandono prematuro de la actividad físico deportiva; y por el contrario, encontró una mayor satisfacción del alumnado con su práctica, cuando esta se relaciona con la complacencia personal en diversos ámbitos dentro del modelo formativo y conlleva a un menor porcentaje de retiro deportivo. Así, el abandono deportivo en las escuelas estudiadas por Nuviala Nuviala, García Montes, Ruiz Juan, \& Jaenes Sánchez, (2006) de carácter competitivo es del $33,6 \%$, mientras que en la escuela de formación deportiva es del $18 \%$.

En el mismo sentido, Jaime Leyva (2010) citado por Villamarin Menza, (2015), encontró que, para la formación de un sistema racional de preparación a largo plazo, se requiere no sólo crear la estructura organizacional (escuela, centro, internado) sino que se necesita considerar diseñar y atender las etapas del proceso de preparación y garantizar los recursos materiales, técnicos y financieros, así como profesionales para desarrollar el sistema: entrenadores, investigadores y profesionales de la salud.

Tal como lo indica Aguirre y Romel (2013) cuando hace referencia a los componentes para una preparación psicológica como: orientaciones de la motivación, rasgos del carácter y temperamento; además muestra un factor importante de que no solamente el entrenador puede dar la motivación psicológica también resalta el apoyo grupal tanto de compañeros como el resto del equipo técnico (medico, fisioterapeuta, preparador físico etc.). (Aguirre, 2013)

En este contexto, concentrar los recursos en los seleccionados nacionales y regionales descuidando el trabajo de las reservas, conduce al desmoronamiento de cualquier sistema de preparación.En consecuencia, estos son los principales problemas que afrontan los clubes y/o escuelas deportivas en correspondencia con la meta de tratar de conformar una masa considerable de deportistas de alto rendimiento y que en su conjunto; son también los problemas que debe afrontar y resolver el Sistema Nacional del Deporte. En una situación de carácter hipotético, el objetivo del sistema o programa nacional de selección y orientación de talentos deportivos, es evitar la pérdida de cientos de niños y niñas con talento para triunfar en el deporte de rendimiento. Se retiran sobre todo al final 
del bachillerato, cuando el cambio de la adolescencia conlleva a los jóvenes a interesarse por otras actividades distintas al deporte, por el paso a la exigencia de la vida universitaria o por la causa más trágica para la sociedad: que no se hayan previsto y dispuesto los medios y estrategias para garantizar la permanencia del niño o joven en el sistema deportivo. Condiciones mínimas de alojamiento, alimentación, salud, educación y por su puesto de procesos adecuados de entrenamiento y competencia para desarrollar su talento. (Villamarin, 2010)

La necesidad de planear e implementar procesos de selección deportiva se debe principalmente a que sólo una pequeña parte de la población que inicia la práctica deportiva llegará a alcanzar altos logros o desempeños deportivos. No todos los sujetos reúnen todas las características genéticas y las condiciones físicas, funcionales y psicológicas necesarias para triunfar. En estudios longitudinales y experiencias desarrolladas en los países socialistas encontraron que de diez mil niños seleccionados después del periodo de enseñanza inicial y que comenzaron los entrenamientos, sólo tres alcanzaron resultados de nivel mundial y olímpico. (Villamarin Menza, 2015)

Por tanto, en la metodología utilizada por los profesores y entrenadores en los procesos de iniciación deportiva suelen existir muchas discrepancias acerca de cuáles son los métodos y procedimientos metodológicos más adecuados y favorables para profesores y alumnos o para deportistas y entrenadores. En general se observa que muchos métodos pueden ser rígidos, escasos de creatividad y preponderantemente empíricos. Un acento en el hacer y el repetir antes que por el analizar y reflexionar sobre la práctica misma. Aunque se aboga por una formación integral en el proceso de preparación deportiva temprana, se termina sobreponiendo el aspecto físico en detrimento de los componentes intelectual y ético.

Sumado a los aspectos familiares, el ambiente en el que se desenvuelven los deportistas y los factores relacionados con los centros de formación; es importante destacar la labor de los técnicos deportivos, los entrenadores, quienes son piezas absolutamente clave en la permanencia del deportista y por ende el desarrollo del alto rendimiento deportivo, ya que son las personas fundamentalmente responsables de que el deportista llegue a generar determinadas actitudes ante el deporte, así como motivar en él emociones y sentimientos de carácter positivo o negativo. De hecho, el carácter autocrático del entrenador da lugar a eventos estresantes muy significativos.

\section{Metodología}

El estudio se adelantó en la ciudad de Bogotá, en las inmediaciones de la pista San Ignacio de la localidad de Kennedy. Desde su enfoque cualitativo atiende de manera transversal los principios bio - éticos autonomía, beneficencia, no maleficencia y justicia con relación a la obligación de respetar los valores y opciones personales de cada individuo que se involucra en la investigación. Este principio constituye el fundamento de la apropiación social del conocimiento y es la regla del consentimiento libre e informado en el que se asume al entrevistado como una persona con capacidad de decidir sobre la información otorgada. Se trata de un estudio de percepción con alcance descriptivo que se enmarca en dos dimensiones de indagación, por un lado, el análisis bibliométrico y documental en cuanto a conocimiento de frontera y por el otro las fuentes primarias de información.

\subsection{Procedimiento}

Encuesta: Padres de familia con hijos patinadores mayores de 12 años, inscritos a clubes de la localidad de Kennedy afiliados a la liga de patinaje de Bogotá.

Población: Finita

Muestra: Probabilística, 94 padres

Nivel de confianza 95\% 
Margen de error: $5 \%$

Recolección de información: Presencial

Sesión de Grupo: Participantes: Focus group de dos horas con 10 Patinadores mayores de 12 años inscritos a clubes de la localidad de Kennedy afiliados a la liga de patinaje de Bogotá. (cinco hombres, cinco mujeres).

Un moderador y dos analistas

La sesión de grupo fue transcrita y analizada por el grupo investigador.

Se solicita autorización a los entrevistados para grabar la sesión, indicándoles que no hay respuestas buenas ni malas, que la información recogida sería únicamente para fines de investigación y requiriendo su autorización para utilizar las opiniones y comentarios que aquí se dieren, en la publicación del estudio.

Entrevistas: Cinco entrevistas: Un entrenador de alto rendimiento, un director de club, un gerente de liga, un presidente de liga y una deportóloga de patinaje.

Cada una de las entrevistas fue transcrita y analizada por el grupo investigador.

Se solicita autorización a los participantes para grabar la entrevista, indicándoles que no hay respuestas buenas ni malas, que la información recogida sería únicamente para fines de investigación y requiriendo su autorización para utilizar las opiniones y comentarios que aquí se dieren, en la publicación del estudio.

Finalmente, se triangula la información resultado de los tres instrumentos para determinar hallazgos y conclusiones.

\section{Resultados}

Los resultados que se presentan a continuación obedecen a la triangulación de la información recogida y analizada por el grupo investigador respecto a cada uno de los instrumentos, teniendo en cuenta la percepción de los diferentes actores que intervienen en la formación y acompañamiento de los patinadores de alto rendimiento y de ellos mismos por lo que resulta pertinente atender y entender las etapas los periodos de crecimiento y maduración de los deportistas.

"Los trabajos que abordan la iniciación a la competición concluyen que el tiempo adecuado puede establecerse operacionalmente e términos del ajuste entre el nivel de crecimiento, madurez y desarrollo, por un lado, y las demandas de la tarea por otro. El crecimiento y la maduración remiten a dimensiones biológicas mientras que el desarrollo incluye a la vez componentes sociales, emocionales, intelectuales y psicomotrices. (Malina, 1986)" (Cantón. E, 1995)

En lo concerniente al factor económico; el estudio evidencia que este item es, tal vez, el de mayor incidencia negativa para que el deportista permanezca en su carrera de alto rendimiento. Vale la pena aclarar que como alto rendimiento se entiende "deporte cuyo objeto es obtener el máximo rendimiento deportivo en competiciones del más alto nivel" (Alonso, 2008) los resultados alcanzados permiten evidenciar que el $85 \%$ de los padres encuestados creen que la economía sí es decisiva a la hora de lograr una continuidad en el proyecto deportivo, consideración que es compartida plenamente por directivos y entrenadores; quienes concuerdan en que el patinaje de carreras es un deporte demasiado costoso; de manera general se aprecia que para un deportista que ya está en competencia y desea sumar en el ranking distrital debe contar con los recursos necesarios para gastos de implementación, nutrición, valoraciones médicas, mensualidades, gimnasios, competencias, hotelería, desplazamientos y más, lo que finalmente termina haciendo mella en el bolsillo de sus principales patrocinadores que, generalmente son los padres del deportista. 
Respecto al factor familia y círculo social; en el primer caso, "Parece evidente que los padres ejercen una influencia determinante sobre varios aspectos del desarrollo de sus hijos, en particular, sobre los aspectos físicos, psicológicos y emocionales." (Piéron, 2007). Esto se va modificando en las diferentes etapas entre la niñez y la adolescencia. En lo relativo al segundo caso, las variables psicológicas pueden ser más importantes durante la adolescencia. el estudio evidencia que definitivamente el círculo social afecta negativamente el progreso del deportista en el alto rendimiento. Las edades donde se comienza a ver afectado el proyecto deportivo son las que van a partir de los 15 años en adelante. Reuniones, compromisos, novio o novia, terminar por distanciar al deportista de su entrenamiento regular. En edades menores (entre 11 y 14 años) el deportista todavía es dependiente de los permisos dados por sus padres, por lo que el círculo social no incide de la misma manera, excepto en los casos en que son los mismos projenitores, por actividades propias de la familia quienes los conducen a interrumir sus compromisos deportivos asumidos con el club.

El $81 \%$ de los padres encuestados creen que son totalmente influyentes en el futuro deportivo de sus hijos, y esto es totalmente acorde a la opinión de deportistas, entrenadores y directivos, quienes encuentran que si no hay un apoyo familiar determinado hacia el deportista, se hace imposible que este puede avanzar en su práctica deportiva.

En cuanto a los elementos asociados con el factor psicológico; el estudio indica que los deportistas de carrera están sometidos de manera permanente a estrés por las altas exigencias al patinador.

“En todas las áreas de funcionamiento que implica el deporte de competición, intervienen variables psicológicas que tienen una notable importancia, como son la motivación, la atención, el estrés, la ansiedad, la autoconfianza, los estados de ánimo, el autocontrol y la autorregulación, la cohesión, las habilidades interpersonales o el ajuste emocional, en la línea señalada por numerosos especialistas (Auweele, De-Cuyper, Van-Mele, y Rzewnicky, 1993; Buceta, 1990 y 1996; García, Rodríguez, Andrade)" (Gimeno. F, 2007)

Cabe resaltar también que los vínculos generados durante la práctica y los lazos de amistad que allí se configuran, afianzan temas relacionados con motivación para continuar. “...uno de los motivos que inducen a la práctica es la búsqueda de reconocimiento social, que produce un aumento significativo de los niveles de AFH (Actividad Física Habitual) en los adolescentes de ambos géneros." (Gálvez \& Velandrino, 2007)

Los resultados de manera particular demuestran, en opinión de los deportistas y los entrenadores que ellos deben aprender a administrar sus éxitos como sus derrotas. Si bien, el $94 \%$ de los padres creen que sus hijos están en condiciones mentales óptimas para afrontar una temporada, la realidad es distinta. En las entrevistas, los deportistas, manifiestan de manera recurrente que se encuentran cansados por las altas exigencias tanto de padres como de entrenadores.

Asociado a lo anterior, y en relación con las condiciones deportivas y del club al que están afiliados los deportistas, se establece por norma que el club debe cumplir con algunos requerimientos básicos que permitan llevar al deportista hacia una formación óptima y de alta competencia; en este sentido, se exige que el club o escuela este "afiliado a su liga respectiva" porque es la única manera de hacer notorio al deportista competitivamente, contar con el escenario indicado y el tiempo suficiente dentro de este.

De igual manera, el club debe contar con entrenadores debidamente capacitados, ya que de esto depende el trabajo deportivo que realizará el patinador, considerando que los procesos no se deben acelerar por dar gusto a las condiciones altamente demandantes del entorno tales como las de de mostrar resultados inmediatos, sin importar edad y proceso del deportista. 
Por último, la gestión técnico-administrativa por parte de los directivos es preponderante, ya que, al ser un deporte tan costoso, el apalancamiento en la empresa privada es necesario para solventar los elevados montos económicos que implica el desarrollo de la temporada.

Entre los diversos factores de influencia producto del estudio que no se encuentran clasificados en los mencionados; se resalta que el factor académico, el cual tiene incidencia significativa en el rendimiento del deportista. En el resultado de las encuestas, especialmente las aplicadas a los padres de familia, se evidencia que el $58 \%$ de los padres creen que el factor académico si afecta la asistencia a los entrenamientos, cotejando estos datos con las respuestas de los deportistas quienes también expresan que el exigente volumen de tareas, sumado a la frecuencia de los entrenamientos, genera cansancio físico, malos resultados y por ende desmotivación.

Aunado a lo anterior, el estudio revela que, sobre los años escolares de décimo y once (grados de la Educación Media, después de la cual se ingresa a las diferentes opciones de la Educación Superior) el deportista comienza a tomar la decisión entre continuar con el proyecto deportivo de rendimiento o dar paso al costado y dedicarse totalmente a sus estudios, pero cada vez la tendencia se va reflejando en edades menores y años escolares inferiores, debido a la necesidad de preparar al deportista a menor edad.

En las entrevistas con directivos, es común encontrar la afirmación: "el patinaje de carreras es un deporte que lo podríamos llamar desagradecido"; para hacer mención a que la inversión tanto en términos económicos como en tiempo, desgaste físico y emocional es muy elevada, y que al final de su carrera deportiva la retribución hacia el patinador es casi nula; lo cual hace que para el patinador sea más rentable tener un proyecto de vida diferente al deportivo, que al paso de los años le dé más réditos y una tranquilidad tanto profesional como económica.

Finalmente, según González (2000), Terribas (2008) y Martínez (2008) la Bioética es la ética de la vida, del ser humano como ser biológico que nace, vive y muere; del ser humano y su entorno social, lo que da origen a derechos y deberes en relación con ese ser viviente. (Molina. V, 2014) Por tanto, la práctica deportiva no es ausente a la relación vital deportista y entorno social.

\section{Conclusiones}

Para que el patinador de carreras alcance el rendimiento deportivo deseado se necesita del trabajo asociado y solidario de tres actores fundamentales: el deportista, el entrenador y el padre de familia. Es importante resaltar estos tres actores como pilares fundamentales del éxito integral del patinador de carreras, a pesar de que el éxito es un concepto demasiado subjetivo y distinto para cada persona; estaría ligado al establecimiento de los objetivos desde el seno del proyecto de cada deportista; por lo cual se espera que tenga claro su proyecto deportivo con objetivos definidos, alcanzables, planificados y medibles, esto le dará cordura al proceso y estabilidad en el progreso.

Para el deportista como actor principal, se espera que él mismo, desde su esencia, se conciba como un deportista de rendimiento, entendiendo que debe tener como meta la obtención de logros que le implicarán a lo largo de su carrera deportiva esfuerzos que otros deportistas "recreativos y formativos" no estarán dispuestos a sobrellevar.

Desde la perspectiva del entorno psicosocial, se puede concluir que no hay un factor dominante que favorezca la continuidad del patinador de carreras. El factor psicológico emerge en las diferentes áreas de su rutina diaria, problemas familiares, estrés académico, acoso escolar por parte de sus compañeros, manejo de lesiones y demás, lo cual hace indispensable enfatizar en la buena preparación mental del deportista, la cual le aportará herramientas para afrontar situaciones adversas o de frustración que tendrá a lo largo de su carrera deportiva. 
En cuanto al factor académico se encontró que tiene gran incidencia sobre los procesos de rendimiento deportivo en el patinador, ya que en su gran mayoría los compromisos académicos restan tiempo para encarar de manera adecuada el rendimiento deportivo. Del mismo modo, se encontró la falta de apoyo desde las instituciones educativas para atender las necesidades del estudiante-deportista, lo que finalmente genera estrés en este y a posteriori el abandono de la práctica deportiva.

Otro factor trascendental, son las dificultades de elección vocacional a futuro, ya que para el patinador de rendimiento se presenta una dicotomía entre dedicarse a su formación académica en una carrera universitaria, o consagrarse exclusivamente al proyecto deportivo posponiendo sus intereses profesionales .

Se encontró que el círculo social va tomando relevancia a medida que el patinador va avanzando en edad, lo que puede ser un distractor e influir en la permanencia en el proyecto deportivo. Si el deportista no se encuentra lo suficientemente motivado hacia su entrenamiento este preferirá realizar otras actividades como, reuniones sociales, salidas recreativas y dedicar tiempo a la pareja, entre otros, los cuales terminarán por alejar al patinador de sus entrenamientos.

La familia y en especial los padres, tienen un papel determinante en el proceso deportivo, entendiendo, que más que un factor son un actor fundamental en la consecución de objetivos del proyecto. La influencia y autoridad que ejercen sobre sus hijos influyen directamente en el avance del patinador en su carrera.

Se encontró, por una parte que cierta lejanía del padre de familia y desconocimiento por parte de la figura paterna en cuanto al papel que cumplen en el proyecto deportivo resulta nociva, siendo necesario el acompañamiento constructivo hacia el proyecto y por otra parte que, la intervención indebida y efusiva puede terminar contrariando y generando conflicto en el contexto del club y por ende en la motivación del patinador.

El factor de mayor influencia en la deserción del deportista, en el cual todos los actores del proceso están de acuerdo; es el factor económico, pues si bien no es un desencadenante directo de deserción, la inversión total marca la diferencia en cuanto a la mejora y continuación del patinador en el rendimiento del deportista.

Otros factores que también influyen sobre la consecución de las metas del patinador de carreras es el estilo de vida y hábitos de los jóvenes deportistas: horas de calidad de sueño, hábitos alimenticios y disciplina en el hogar, que definitivamente si no se manejan de la manera correcta influirán sobre el rendimiento y avance del deportista.

A propósito de las características asociadas al club / escuela, para favorecer la permanencia de sus patinadores a partir de los 12 años de edad; se concluye que definitivamente es el club el responsable de definir la clase de proyecto que el deportista debe asumir a lo largo del camino hacia el rendimiento, y éste desde su dirigencia, es responsable de impartir la visión tanto a padres de familia como a deportistas, realizando un trabajo mancomunado en búsqueda del cumplimiento de los objetivos propuestos, entendiendo que es el club en cabeza de directivos y entrenadores quien debe liderar los procesos.

En lo que concierne a la gerencia del club, se debe realizar una planeación adecuada teniendo objetivos claros con el fin de que exista un manejo apropiado del recurso, dando cumplimiento a lo presupuestado. La realidad que enmarca a una gran mayoría de clubes es el poco interés que se está dando a la parte administrativa, lo cual no permite el fortalecimiento de las instituciones y en consecuencia de sus deportistas.

El proyecto deportivo del club es la esencia del rendimiento deportivo, por lo que este debe ser un trazado profesional, pensando en las cargas aplicadas a los deportistas, y no solo en resultados inmediatos. Siendo coherente con este punto, para poder llevar a flote un buen proyecto deportivo el club también debe contar con personal idóneo y capacitado; pues son los entrenadores los que directamente tienen la responsabilidad de 
transmitir el conocimiento a sus deportistas. El papel del entrenador dentro del proyecto es muy importante ya que es el encargado de transmitir, influir y motivar a sus aprendices; es quien de primera mano se relaciona con los deportistas, conociendo tanto sus frustraciones como sus logros. Por tanto, gran parte de la motivación del deportista pasa por las estrategias que tienen los docentes para lograr apasionar al patinador por su deporte.

En correspondencia, es fundamental que dentro de las estimaciones del club, tanto deportistas como padres se vean beneficiados por la acción de un equipo interdisciplinario conformado idealmente por psicólogo, médico deportivo, nutricionista y fisioterapeuta. Este equipo posibilita la solución integrada de problemas que puedan afrontar los deportistas. Si bien, la mayoría de los directores entrevistados aducen el no poder contar con los servicios por concepto de los altos costos, si se puede establecer alianzas profesionales con centros médicos o acuerdos entre los mismos clubes con el fin de reducir costos.

Definitivamente, un patinador de rendimiento debe entrenar en los espacios adecuados para ello, se hace necesario que el club gestione escenarios que cuenten con la seguridad, medidas y tiempo de uso reglamentario para realizar buenas prácticas. Así mismo, planificar un cronograma de manera adecuada pensando en el deportista, fechas, distancias, horas y lugares en los que estos puedan realizar sus entrenamientos sin contratiempos.

Otro factor de vital importancia es el compromiso ético de los directivos y demás involucrados que dan curso al proyecto deportivo dentro de cada entidad. Criterios éticos rigurosos deben servir para apoyar y alentar a los patinadores de carrera, entendiendo que el deporte como tal, debe ser una herramienta para el enriquecimiento integral del Ser y no un fin para la explotación física y deportiva del atleta. Por el contrario, el patinaje deber ser un medio por el cual se hace una construcción de un Ser humano positivo y un ciudadano referente para la juventud y la sociedad.

Si bien es cierto que el buen nombre de cada club está relacionado con sus buenos resultados deportivos, este no debe entrar en una carrera desmedida por la obtención de medallas, dejando a un lado al deportista como ser físico, afectivo, y espiritual. La responsabilidad del club debe ser la de generar iniciativas que concedan la importancia de la ética deportiva tomando el liderazgo en cuanto a la concienciación de adecuados procesos con los otros actores, padres de familia y deportistas.

\section{Referencias bibliográficas}

Aguirre, A. y. (2013). Aplicación de un protocolo de intervención psicológica para las competencias en los deportistas seleccionados de Tae Kwon Do de la categoría juvenil de Concentración Deportiva de Pichincha. Obtenido de www.dspace.uce.edu.ec: http://www.dspace.uce.edu.ec/bitstream/25000/1354/1/T-UCE-0007-15.pdf

Cantón, E. (1995). Factores motivacionales y afectivos en la iniciación deportiva. Revista de Psicología General y Aplicada, 59-75.

Gálvez, A., \& Velandrino, A. (2007). Influencia de determinados motivos de práctiva físico-deportiva sobre los niveles de actividad física habitual en los adolescentes . Cuadernos de Psicología del Deporte, 7, 71-84.

Gimeno, F. (2007). Influencia de las variables psicológicas en el deporte de competición: evaluación mediante el cuestionario Características psicológicas relacionadas con el rendimiento deportivo. (U. d. Oviedo, Ed.) Psicothema, vol. 19(4), 667-672.

IDRD. (31 de Agosto de 2020). https://www.idrd.gov.co. Obtenido de https://www.idrd.gov.co: https://www.idrd.gov.co/escuelas-avaladas 
Macarro Moreno, J. R. (2010). Motivos de abandono de la práctica de actividad físico-deportiva en los estudiantes de bachillerato de la provincia de Granada. Revista de Educación, 495-519.

Molina, V. (2014). Bioética: discapacidad y actividad física. Revista Ciencias de la Actividad Físicca, 15(2), 78-85.

Piéron, M. (2007). Factores determinantes en la inactividad físico-deportiva en jóvenes y adolescentes. VII Congreso Internacional sobre la Enseñanza de la Educación física y el Deporte escolar. (págs. 15-67). Badajoz: FEADEF y AMEFEX. Recuperado el 2020

Esta obra está bajo una Licencia Creative Commons Attribución-NoCommercial 4.0 International

\section{(cc) BY-NG}

